International Journal of Pure and Applied Mathematics

Volume 104 No. 1 2015, 29-42

ISSN: 1311-8080 (printed version); ISSN: 1314-3395 (on-line version)

url: http://www.ijpam.eu

doi: http://dx.doi.org/10.12732/ijpam.v104i1.3

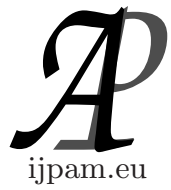

\title{
STATISTICAL CONVERGENCE \\ IN FUZZY $n$-NORMED SPACES
}

\author{
B. Surender Reddy ${ }^{1}$, M. Srinivas ${ }^{2} \S$ \\ ${ }^{1,2}$ Department of Mathematics \\ University College of Science \\ Saifabad, Osmania University \\ Hyderabad, Telangana 500004, INDIA
}

\begin{abstract}
In this paper we introduce the concept of statistical convergence of a sequence in fuzzy $n$-normed space and defined limit point, statistical limit point and statistical cluster point of a sequence in fuzzy $n$-normed space. Also we establish the relationship between limit point, statistical limit point and statistical cluster point of a sequence in fuzzy $n$-normed space.
\end{abstract}

AMS Subject Classification: 46S40, 41A50

Key Words: fuzzy $n$-normed space, statistical convergence and statistical Cauchy sequence in fuzzy $n$-normed Space, statistical limit point and statistical cluster point in fuzzy n-normed space

\section{Introduction and Preliminaries}

The idea of fuzzy norm on a vector space was initiated by Katsaras [15] in [1984]. Later Felbin [17] introduced the concept of fuzzy normed space in [1992] which is based on the concept of fuzzy metric given by Kaleva and Siekkala [16]. They

Received: March 24, 2015

(C) 2015 Academic Publications, Ltd. url: www.acadpubl.eu

${ }^{\S}$ Correspondence author 
also defined fuzzy bounded linear operator and norm of which is a fuzzy number. In [2003] Bag and Samanta [1] discussed some of the properties of finite dimensional fuzzy normed linear spaces. In [1979] Mizumoto and Tanaka [5] defined fuzzy number and discussed some of the properties of fuzzy numbers. The theory of fuzzy $n$-normed spaces was studied by Narayanan and Vijayabalaji [18].

The idea of statistical convergence was given by Steinhaus [7] in [1951]. Fridy [14] developed the idea of statistical limit point, statistical cluster point of a number sequence. In [2010] B.S.Reddy [10] studied statistical convergence in $n$-normed space and some results are established.

Statistical convergence applied in many branches of mathematics like trigonometric series, number theory and summability theory [8]. The concept of statistical convergence in fuzzy normed space was given by Sencimen, Pehlivan [4] later Mohiuddine, Sevli and Cancan [12] extended the concept of statistical convergence in fuzzy normed space to statistical convergence in fuzzy 2-normed space.

In this paper we introduce the concept of statistical convergence of a sequence in fuzzy $n$-normed space and defined limit point, statistical limit point and statistical cluster point of a sequence in fuzzy $n$-normed space. Also we establish the relationship between limit point, statistical limit point and statistical cluster point of a sequence in fuzzy $n$-normed space.

According to Mizumoto and Tanaka [5], a fuzzy number is a mapping $x$ : $R \longrightarrow[0,1]$ over the set $R$ of real numbers. A fuzzy number $x$ is said to be convex if for any real numbers $t, s, r \in R$ with $s \leq t \leq r, x(t) \geq \min \{x(s), x(r)\}$. A fuzzy number $x$ is said to be normal if $\exists t_{0} \in R$ such that $x\left(t_{0}\right)=1$. For $0<\alpha \leq 1, \alpha-$ Level set of an upper semi continuous convex normal fuzzy number $\eta$ (denoted by $[\eta]_{\alpha}$ ) is a closed interval $\left[a_{\alpha}, b_{\alpha}\right]$, where $a_{\alpha}=-\infty$ and $b_{\alpha}=+\infty$ admissible. When $a_{\alpha}=-\infty$, for instance, then $\left[a_{\alpha}, b_{\alpha}\right]$ means the interval $\left(-\infty, b_{\alpha}\right]$. Similarly when $b_{\alpha}=+\infty$ then $\left[a_{\alpha}, b_{\alpha}\right]$ means the interval $\left[a_{\alpha},+\infty\right)$. A fuzzy number $x$ is said to be non-negative if $x(t)=0, \forall t<0$. The set of all convex, normal, upper semi continuous fuzzy real numbers is denoted by $L(R)$ and the set of all non-negative, convex, normal, upper semi continuous fuzzy real numbers is denoted by $L^{*}(R)$.

For any real number $r \in R$, we define a corresponding fuzzy number $\hat{r}$ by

$$
\begin{aligned}
\hat{r} & =1 \text { if } t=r \\
& =0 \text { if } t \neq r
\end{aligned}
$$

A partial ordering $\preceq$ on $L(R)$ is defined by $u \preceq v$ if and only if $u_{\alpha}^{-} \leq v_{\alpha}^{-}$ and $u_{\alpha}^{+} \leq v_{\alpha}^{+} \forall \alpha \in[0,1]$, where $[u]_{\alpha}=\left[u_{\alpha}^{-}, u_{\alpha}^{+}\right]$and $[v]_{\alpha}=\left[v_{\alpha}^{-}, v_{\alpha}^{+}\right]$. The strict 
inequality in $L(R)$ is defined by $u \prec v$ if and only if $u_{\alpha}^{-}<v_{\alpha}^{-}$and $u_{\alpha}^{+}<v_{\alpha}^{+}$ $\forall \alpha \in[0,1]$. For $k>0,(k u)$ is defined as $(k u)(t)=u(t / k)$ and $(0 u)(t)=u(t / k)$ is defined to be $\hat{0}(t)$.

According to Mizumoto and Tanaka [5], the arithmetic operations $\oplus, \ominus, \otimes$ on $L(R) \times L(R)$ are defined by

$$
\begin{aligned}
& (x \oplus y)(t)=\sup _{s \in R} \min \{x(s), y(t-s)\} \\
& (x \ominus y)(t)=\sup _{s \in R} \min \{x(s), y(s-t)\}
\end{aligned}
$$

and

$$
(x \otimes y)(t)=\sup _{s \in R, s \neq 0} \min \{x(s), y(t / s)\}, \quad \forall t \in R
$$

Let $u, v \in L(R)$. Define $D(u, v)=\sup _{\alpha \in[0,1]} \max \left\{\left|u_{\alpha}^{-}-v_{\alpha}^{-}\right|,\left|u_{\alpha}^{+}-v_{\alpha}^{+}\right|\right\}$Then $D$ is called supremum metric on $L(R)$. Let $\left(u_{n}\right) \subset L(R)$ and $u \in L(R)$. A sequence $\left(u_{n}\right)$ is said to be converges to $u$ in the metric $D$ if $\lim _{n \rightarrow \infty} D\left(u_{n}, u\right)=0$ (for short, $D$-converges to $u$ ), It can be written as $u_{n} \longrightarrow^{D} u$ or $(D)-\lim _{n \rightarrow \infty} u_{n}=u$.

\section{Fuzzy $n$-Normed Spaces}

Definition 1. Let $n \in N$ and let $X$ be a real linear space of dimension $d \geq n$. A real valued function $\|\bullet, \bullet, \ldots, \bullet\|$ on $\underbrace{X \times X \times \cdots \times X}_{n}=X^{n} \longrightarrow R^{n}$ satisfying the following conditions

$n N_{1}: \quad\left\|x_{1}, x_{2}, \ldots, x_{n}\right\|=0$ if and only if $x_{1}, x_{2}, \ldots, x_{n}$ are linearly dependent

$n N_{2}: \quad\left\|x_{1}, x_{2}, \ldots, x_{n}\right\|$ is invariant under any permutation of $x_{1}, x_{2}, \ldots, x_{n}$

$n N_{3}: \quad\left\|x_{1}, x_{2}, \ldots, x_{n-1}, \alpha x_{n}\right\|=|\alpha|\left\|x_{1}, x_{2}, \ldots, x_{n}\right\|$ for all $\alpha \in R$

$n N_{4}: \quad\left\|x_{1}, x_{2}, \ldots, x_{n-1}, y+z\right\| \leq\left\|x_{1}, x_{2}, \ldots, x_{n-1}, y\right\|+\left\|x_{1}, x_{2}, \ldots, x_{n-1}, z\right\|$ for all $y, z, x_{1}, x_{2}, \ldots, x_{n-1} \in X$, then the function $\|\bullet, \bullet, \ldots, \bullet\|$ is called an $n$ norm on $X$ and the pair $(X,\|\bullet, \bullet, \ldots, \bullet\|)$ is called $n$-normed linear space.

Definition 2. Let $X$ be a real linear space of dimension $d$, where $2 \leq$ $d<\infty$. Let $\|\bullet, \bullet, \ldots, \bullet\|: \underbrace{X \times X \times \cdots \times X}_{n} \longrightarrow L^{*}(R)$ and the mappings $L, R$ : $[0,1] \times[0,1] \longrightarrow[0,1]$ be symmetric, non-decreasing in both arguments such that $L(0,0)=0, R(1,1)=1$ then the quadruple $(X,\|\bullet, \bullet, \ldots, \bullet\|, L, R)$ is called fuzzy $n$-normed space (in short $\mathrm{f}-n$-N) if it satisfies the following conditions for every $x_{1}, x_{2}, \ldots, x_{n}, y \in X$ and $s, t \in R$ 
$\left(f-n N_{1}\right):\left\|x_{1}, x_{2}, \ldots, x_{n}\right\|=\overline{0}$ if and only if $x_{1}, x_{2}, \ldots, x_{n}$ are linearly dependent vectors,

$\left(f-n N_{2}\right):\left\|x_{1}, x_{2}, \ldots, x_{n}\right\|$ is invariant under any permutation of $x_{1}, x_{2}, \ldots$, $x_{n}$,

$\left(f-n N_{3}\right):\left\|\alpha x_{1}, x_{2}, \ldots, x_{n}\right\|=|\alpha|\left\|x_{1}, x_{2}, \ldots, x_{n}\right\|, \quad \alpha \in R$

$\left(f-n N_{4}\right):\left\|x_{1}+y, x_{2}, \ldots, x_{n}\right\|(s+t) \geq L\left(\left\|x_{1}, x_{2}, \ldots, x_{n}\right\|(s), \| y, x_{2}, \ldots\right.$, $\left.x_{n} \|(t)\right)$, whenever $s \leq\left\|x_{1}, x_{2}, \ldots, x_{n}\right\|_{1}^{-}, \quad t \leq\left\|y, x_{2}, \ldots, x_{n}\right\|_{1}^{-}$and $s+t \leq$ $\left\|x_{1}+y, x_{2}, \ldots, x_{n}\right\|_{1}^{-}$,

$\left(f-n N_{5}\right):\left\|x_{1}+y, x_{2}, \ldots, x_{n}\right\|(s+t) \leq R\left(\left\|x_{1}, x_{2}, \ldots, x_{n}\right\|(s), \| y, x_{2}, \ldots\right.$, $\left.x_{n} \|(t)\right)$, whenever $s \geq\left\|x_{1}, x_{2}, \ldots, x_{n}\right\|_{1}^{-}, t \geq\left\|y, x_{2}, \ldots, x_{n}\right\|_{1}^{-}$and $s+t \geq$ $\left\|x_{1}+y, x_{2}, \ldots, x_{n}\right\|_{1}^{-}$,

where $\left[\left\|x_{1}, x_{2}, \ldots, x_{n}\right\|\right]_{\alpha}=\left[\left\|x_{1}, x_{2}, \ldots, x_{n}\right\|_{\alpha}^{-},\left\|x_{1}, x_{2}, \ldots, x_{n}\right\|_{\alpha}^{+}\right]$for $x_{1}, x_{2}, \ldots, x_{n} \in X, 0 \leq \alpha \leq 1$ and $\inf _{\alpha \in[0,1]}\left\|x_{1}, x_{2}, \ldots, x_{n}\right\|_{\alpha}^{-}>0$. Hence the norm $\|\bullet, \bullet, \ldots, \bullet\|$ is called fuzzy $n$-norm.

Let us consider the topological structure of fuzzy $n$-normed space $(X, \| \bullet, \bullet$, $\ldots, \bullet \|, L, R)$. For any $\epsilon>0, \alpha \in[0.1]$ and $x \in X$, the $(\epsilon, \alpha)$-neighborhood of $x$ is the set $N_{x}(\epsilon, \alpha)=\left\{y \in X:\left\|x-y, z_{2}, z_{3}, \ldots, z_{n}\right\|_{\alpha}^{+}<\epsilon\right\}$ for each $z_{2}, z_{3}, \ldots, z_{n} \neq 0, z_{2}, z_{3}, \ldots, z_{n} \in X$.

Definition 3. A sequence $\left\{x_{k}\right\}$ in fuzzy n-normed space $(X,\|\bullet, \bullet, \ldots, \bullet\|$, $L, R)$ is said to be convergent to an element $x \in X$ with respect to the fuzzy $n$-norm on $X$ if for every $\epsilon>0$ and for every $z_{2}, z_{3}, \ldots, z_{n} \neq 0, z_{2}, z_{3}, \ldots, z_{n} \in X$, $\exists$ a number $N=N\left(\epsilon, z_{2}, z_{3}, \ldots, z_{n}\right)$ such that $D\left(\left\|x_{k}-x, z_{2}, z_{3}, \ldots, z_{n}\right\|, \overline{0}\right)<\epsilon$, $\forall k \geq N$ or equivalently $(D)-\lim _{k \rightarrow \infty}\left\|x_{k}-x, z_{2}, z_{3}, \ldots, z_{n}\right\|=\overline{0}$.

It can be represented by $\left(x_{k}, z_{2}, z_{3}, \ldots, z_{n}\right) \rightarrow^{f-n-N}\left(x, z_{2}, z_{3}, \ldots, z_{n}\right)$ for every $z_{2}, z_{3}, \ldots, z_{n} \neq 0, z_{2}, z_{3}, \ldots, z_{n} \in X$

This means that for every $\epsilon>0, \exists$ a number $N=N\left(\epsilon, z_{2}, z_{3}, \ldots, z_{n}\right)$ such that $\sup _{\alpha \in[0,1]}\left\|x_{k}-x, z_{2}, z_{3}, \ldots, z_{n}\right\|_{\alpha}^{+}=\left\|x_{k}-x, z_{2}, z_{3}, \ldots, z_{n}\right\|_{0}^{+}<\epsilon \quad \forall k \geq N$. $\alpha \in[0,1]$

In terms of neighborhood, we have $\left(x_{k}, z_{2}, z_{3}, \ldots, z_{n}\right) \longrightarrow^{f-n-N}\left(x, z_{2}, z_{3}, \ldots, z_{n}\right)$ if for any $\epsilon>0, \exists$ a number $N=N\left(\epsilon, z_{2}, z_{3}, \ldots, z_{n}\right)$ such that $x_{k} \in N_{x}(\epsilon, 0)$, whenever $k \geq N$.

\section{Statistical Convergence and Statistically Cauchy Sequences in Fuzzy $n$-Normed Spaces}

In this section, we define the notion of statistically convergence and statistically Cauchy sequences in fuzzy $n$-normed space. 
Let $K$ be a subset of natural numbers $N$. Then the natural density of $K$ denoted by $\delta(K)$, is defined as $\delta(K)=\lim _{n} \frac{1}{n}|\{k \leq n: k \in K\}|$, where the vertical bars denote the cardinality of the enclosed set.

A number sequence $\left\{x_{k}\right\}$ is said to be statistically convergent to the number $\ell$ if for each $\epsilon>0$, the set $K(\epsilon)=\left\{k \leq n: \| x_{k}-\ell \mid \geq \epsilon\right\}$ has natural density zero. i.e., $\lim _{n} \frac{1}{n}\left|\left\{k \leq n:\left|x_{k}-\ell\right| \geq \epsilon\right\}\right|=0$. In this case we write $s t-\lim x_{k}=\ell$. Every convergent sequence is statistically convergent to the same limit, but the converse need not be true.

Statistical convergence in $n$-normed space has been studied by B.S. Reddy in [10].

Let $\left\{x_{k}\right\}$ be a sequence in $n$-normed space $(X,\|\bullet, \bullet, \ldots, \bullet\|)$. The sequence $\left\{x_{k}\right\}$ is said to be statistically convergent to $\ell$, if for every $\epsilon>0$, the set $\{k \in$ $\left.N:\left\|x_{k}-\ell, z_{2}, z_{3}, \ldots, z_{n}\right\| \geq \epsilon\right\}$ has natural density zero for each $z_{2}, z_{3}, \ldots, z_{n} \neq 0$, $z_{2}, z_{3}, \ldots, z_{n} \in X$. In other words $\left\{x_{k}\right\}$ statistically converges to $\ell$ in $n$-normed space $(X,\|\bullet, \bullet, \ldots, \bullet\|)$ if $\lim _{m \rightarrow \infty} \frac{1}{m}\left|\left\{k \leq m:\left\|x_{k}-\ell, z_{2}, z_{3}, \ldots, z_{n}\right\| \geq \epsilon\right\}\right|=0$ for each $z_{2}, z_{3}, \ldots, z_{n} \neq 0, z_{2}, z_{3}, \ldots, z_{n} \in X$. It means that for every $z_{2}, z_{3}, \ldots, z_{n} \in X$ we have $\left\|x_{k}-\ell, z_{2}, z_{3}, \ldots, z_{n}\right\|<\epsilon$ for almost all $k$ (for short a.a.k). In this case we write $s t-\lim _{k \rightarrow \infty}\left\|x_{k}, z_{2}, z_{3}, \ldots, z_{n}\right\|=\left\|\ell, z_{2}, z_{3}, \ldots, z_{n}\right\|$.

Definition 4. Let $\left\{x_{k}\right\}$ be a sequence in fuzzy $n$-normed space $(X, \| \bullet, \bullet$ $\ldots, \bullet \|, L, R)$. Then the sequence $\left\{x_{k}\right\}$ is said to be statistically convergent to an element $x \in X$ with respect to the fuzzy $n$-norm on $X$ if for every $\epsilon>0$ and every $z_{2}, z_{3}, \ldots, z_{n} \neq 0, z_{2}, z_{3}, \ldots, z_{n} \in X$ we have $\delta(\{k \in N$ : $\left.\left.D\left(\left\|x_{k}-x, z_{2}, z_{3}, \ldots, z_{n}\right\|, \overline{0}\right) \geq \epsilon\right\}\right)=0$ or equivalently $\delta\left(\left\{k \in N: D\left(\| x_{k}-\right.\right.\right.$ $\left.\left.\left.x, z_{2}, z_{3}, \ldots, z_{n} \|, \overline{0}\right)<\epsilon\right\}\right)=1$. Which gives that for each $\epsilon>0$ and $z_{2}, z_{3}, \ldots, z_{n} \in$ $X$, the set $K(\epsilon)=\left\{k \in N:\left\|x_{k}-x, z_{2}, z_{3}, \ldots, z_{n}\right\|_{0}^{+} \geq \epsilon\right\}$ has natural density zero; namely for each $\epsilon>0,\left\|x_{k}-x, z_{2}, z_{3}, \ldots, z_{n}\right\|_{0}^{+}<\epsilon$ for almost all $k$. In this case we write $s t(f-n-N)-\lim _{k \rightarrow \infty}\left\|x_{k}-x, z_{2}, z_{3}, \ldots, z_{n}\right\|=0$ either $s t-\lim _{k \rightarrow \infty}\left\|x_{k}-x, z_{2}, z_{3}, \ldots, z_{n}\right\|=0$ or $\left(x_{k}, z_{2}, z_{3}, \ldots, z_{n}\right) \longrightarrow^{s t(f-n-N)}$ $\left(x, z_{2}, z_{3}, \ldots, z_{n}\right)$.

In terms of neighborhoods, we have

$$
\left(x_{k}, z_{2}, z_{3}, \ldots, z_{n}\right) \longrightarrow^{s t(f-n-N)}\left(x, z_{2}, z_{3}, \ldots, z_{n}\right)
$$

if for every $\epsilon>0, \delta\left(\left\{k \in N: x_{k} \notin N_{x}(\epsilon, 0)\right\}\right)=0$, i.e., for each $\epsilon>0$, $\left\{x_{k}\right\} \in N_{x}(\epsilon, 0)$ for almost all $k$.

A useful interpretation of the above definition is the following:

$$
\left(x_{k}, z_{2}, z_{3}, \ldots, z_{n}\right) \longrightarrow^{s t(f-n-N)}\left(x, z_{2}, z_{3}, \ldots, z_{n}\right)
$$


iff $s t-\lim _{k \rightarrow \infty}\left\|x_{k}-x, z_{2}, z_{3}, \ldots, z_{n}\right\|_{0}^{+}=0$. Note that $s t-\lim _{k \rightarrow \infty}\left\|x_{k}-x, z_{2}, z_{3}, \ldots, z_{n}\right\|_{0}^{+}=$ 0 implies that $s t-\lim _{k \rightarrow \infty}\left\|x_{k}-x, z_{2}, z_{3}, \ldots, z_{n}\right\|_{\alpha}^{-}=s t-\lim _{k \rightarrow \infty}\left\|x_{k}-x, z_{2}, z_{3}, \ldots, z_{n}\right\|_{\alpha}^{+}=$ 0 for each $\alpha \in[0,1]$ since $0 \leq\left\|x_{k}-x, z_{2}, z_{3}, \ldots, z_{n}\right\|_{\alpha}^{-} \leq\left\|x_{k}-x, z_{2}, z_{3}, \ldots, z_{n}\right\|_{\alpha}^{+} \leq$ $\left\|x_{k}-x, z_{2}, z_{3}, \ldots, z_{n}\right\|_{0}^{+}$holds for every $k \in N$ and for each $\alpha \in[0,1]$. Hence the result.

Definition 5. Let $\left\{x_{k}\right\}$ be a sequence in fuzzy $n$-normed space $(X, \| \bullet, \bullet$, $\ldots, \bullet \|, L, R)$. Then the sequence $\left\{x_{k}\right\}$ is said to be statistically Cauchy sequence with respect to the fuzzy $n$-norm on $X$ if for every $\epsilon>0, \exists$ a number $N=$ $N\left(\epsilon, z_{2}, z_{3}, \ldots, z_{n}\right)$ such that $\delta\left(\left\{k \in N:\left\|x_{k}-x_{N\left(\epsilon, z_{2}, z_{3}, \ldots, z_{n}\right)}, z_{2}, z_{3}, \ldots, z_{n}\right\|_{0}^{+} \geq\right.\right.$ $\epsilon\})=0$.

Theorem 6. Let $\left\{x_{k}\right\},\left\{y_{k}\right\}$ be two sequences in fuzzy n-normed space $(X,\|\bullet, \bullet, \ldots, \bullet\|, L, R)$ such that

$$
\left(x_{k}, z_{2}, z_{3}, \ldots, z_{n}\right) \longrightarrow{ }^{s t(f-n-N)}\left(x, z_{2}, z_{3}, \ldots, z_{n}\right)
$$

and

$$
\left(y_{k}, z_{2}, z_{3}, \ldots, z_{n}\right) \longrightarrow^{s t(f-n-N)}\left(y, z_{2}, z_{3}, \ldots, z_{n}\right)
$$

for all $x, y \in X$ and $z_{2}, z_{3}, \ldots, z_{n} \neq 0, z_{2}, z_{3}, \ldots, z_{n} \in X$ then:

(i) $\left(x_{k}+y_{k}, z_{2}, z_{3}, \ldots, z_{n}\right) \longrightarrow^{s t(f-n-N)}\left(x+y, z_{2}, z_{3}, \ldots, z_{n}\right)$.

(ii) $\left(\alpha x_{k}, z_{2}, z_{3}, \ldots, z_{n}\right) \longrightarrow{ }^{s t(f-n-N)}\left(\alpha x, z_{2}, z_{3}, \ldots, z_{n}\right)$.

(iii) $\operatorname{st}(f-n-N)-\left\|\left(x_{k}, z_{2}, z_{3}, \ldots, z_{n}\right)=\right\|\left(x, z_{2}, z_{3}, \ldots, z_{n}\right) \|$.

Proof. (i) Let us assume that

$$
\left(x_{k}, z_{2}, z_{3}, \ldots, z_{n}\right) \longrightarrow^{s t(f-n-N)}\left(x, z_{2}, z_{3}, \ldots, z_{n}\right)
$$

and

$$
\left(y_{k}, z_{2}, z_{3}, \ldots, z_{n}\right) \longrightarrow^{s t(f-n-N)}\left(y, z_{2}, z_{3}, \ldots, z_{n}\right) .
$$

Since $\|\bullet, \bullet, \ldots, \bullet\|_{0}^{+}$is a $n$-norm in the usual sense, we get

$$
\begin{aligned}
\left\|\left(x_{k}+y_{k}\right)-(x+y), z_{2}, z_{3}, \ldots, z_{n}\right\|_{0}^{+} \leq\left\|x_{k}-x, z_{2}, z_{3}, \ldots, z_{n}\right\|_{0}^{+} & \\
& +\left\|y_{k}-y, z_{2}, z_{3}, \ldots, z_{n}\right\|_{0}^{+},
\end{aligned}
$$

for all $k \in N$ and every $z_{2}, z_{3}, \ldots, z_{n} \neq 0, z_{2}, z_{3}, \ldots, z_{n} \in X$. Setting

$$
\begin{aligned}
K(\epsilon) & =\left\{k \in N:\left\|\left(x_{k}+y_{k}\right)-(x+y), z_{2}, z_{3}, \ldots, z_{n}\right\|_{0}^{+} \geq \epsilon\right\}, \\
K_{1}(\epsilon) & =\left\{k \in N:\left\|x_{k}-x, z_{2}, z_{3}, \ldots, z_{n}\right\|_{0}^{+} \geq \frac{\epsilon}{2}\right\},
\end{aligned}
$$




$$
K_{2}(\epsilon)=\left\{k \in N:\left\|y_{k}-y, z_{2}, z_{3}, \ldots, z_{n}\right\|_{0}^{+} \geq \frac{\epsilon}{2}\right\} .
$$

From (1) we have $K(\epsilon) \subseteq K_{1}(\epsilon) \cup K_{2}(\epsilon)$. Now by our assumption $\delta\left(K_{1}(\epsilon)\right)=$ $\delta\left(K_{2}(\epsilon)\right)=0$. Which gives $\delta(K(\epsilon))=0$, i.e.

$$
\left(x_{k}+y_{k}, z_{2}, z_{3}, \ldots, z_{n}\right) \longrightarrow^{s t(f-n-N)}\left(x+y, z_{2}, z_{3}, \ldots, z_{n}\right) .
$$

(ii) This proof is very simple.

(iii) Since $\|\bullet, \bullet, \ldots, \bullet\|_{\alpha}^{-}$and $\|\bullet \bullet \bullet, \ldots, \bullet\|_{\alpha}^{+}$are $n$-norms in the usual sense, we have

$$
0 \leq\left|\left\|x_{k}, z_{2}, z_{3}, \ldots, z_{n}\right\|_{\alpha}^{-}-\left\|x, z_{2}, z_{3}, \ldots, z_{n}\right\|_{\alpha}^{-}\right| \leq\left\|x_{k}-x, z_{2}, z_{3}, \ldots, z_{n}\right\|_{\alpha}^{-}
$$

and

$$
0 \leq\left|\left\|x_{k}, z_{2}, z_{3}, \ldots, z_{n}\right\|_{\alpha}^{+}-\left\|x, z_{2}, z_{3}, \ldots, z_{n}\right\|_{\alpha}^{+}\right| \leq\left\|x_{k}-x, z_{2}, z_{3}, \ldots, z_{n}\right\|_{\alpha}^{+},
$$

for all $\alpha \in[0,1]$. Therefore

$$
\begin{array}{r}
0 \leq \max \left\{\left|\left\|x_{k}, z_{2}, z_{3}, \ldots, z_{n}\right\|_{\alpha}^{-}-\left\|x, z_{2}, z_{3}, \ldots, z_{n}\right\|_{\alpha}^{-}\right|, \mid\left\|x_{k}, z_{2}, z_{3}, \ldots, z_{n}\right\|_{\alpha}^{+}\right. \\
\left.-\left\|x, z_{2}, z_{3}, \ldots, z_{n}\right\|_{\alpha}^{+} \mid\right\} \leq\left\|x_{k}-x, z_{2}, z_{3}, \ldots, z_{n}\right\|_{\alpha}^{+}
\end{array}
$$

for all $\alpha \in[0,1]$. Taking supremum over $\alpha \in[0,1]$, we get

$0 \leq D\left(\left\|x_{k}, z_{2}, z_{3}, \ldots, z_{n}\right\|,\left\|x, z_{2}, z_{3}, \ldots, z_{n}\right\|\right) \leq\left\|x_{k}-x, z_{2}, z_{3}, \ldots, z_{n}\right\|_{0}^{+}$. Hence $\operatorname{st}(f-n-N)-\left\|\left(x_{k}, z_{2}, z_{3}, \ldots, z_{n}\right)\right\|=\left\|\left(x, z_{2}, z_{3}, \ldots, z_{n}\right)\right\|$.

Lemma 7. Let $\left\{A_{i}: i \in I\right\}$ be a countable collection of subset of $N$ such that $\delta\left(A_{i}\right)=1$ for each $i \in I$. then there is a set $A \subset N$ such that $\delta(A)=1$ and $\left|A \backslash A_{i}\right|<\infty$ for all $i \in I$.

Theorem 8. Let $\left\{x_{k}\right\}$ be a sequence in fuzzy n-normed space $(X, \| \bullet, \bullet, \ldots$, $\bullet \|, L, R)$. Then $\left\{x_{k}\right\}$ is statistically convergent to an element $x \in X$ with respect to the fuzzy n-norm on $X$ if and only if $\left\{x_{k}\right\}$ is a sequence for which there is a sequence $\left\{y_{k}\right\}$ that is convergent such that $x_{k}=y_{k}$ for a.a.k.

Proof. Let us assume that $\left(x_{k}, z_{2}, z_{3}, \ldots, z_{n}\right) \longrightarrow^{s t(f-n-N)}\left(x, z_{2}, z_{3}, \ldots, z_{n}\right)$. For each $i \in N$, let $A_{i}=\left\{k \in N:\left\|\left(x_{k}-x, z_{2}, z_{3}, \ldots, z_{n}\right)\right\|_{0}^{+} \leq \frac{1}{i}\right\}$ and $\delta\left(A_{i}\right)=1$ for each i, since $\left(x_{k}, z_{2}, z_{3}, \ldots, z_{n}\right) \longrightarrow^{s t(f-n-N)}\left(x, z_{2}, z_{3}, \ldots, z_{n}\right)$. Let $A$ be as given in Lemma 3.4. For every $\epsilon>0, \exists$ a number $N=N\left(\epsilon, z_{2}, z_{3}, \ldots, z_{n}\right)$ such that $k \geq N$ and $k \in A$ imply $\left\|\left(x_{k}-x, z_{2}, z_{3}, \ldots, z_{n}\right)\right\|_{0}^{+}<\epsilon$. Define a sequence $\left\{y_{k}\right\}$ as $y_{k}=x_{k}$ for each $k \in A$ and $y_{k}=x$ for $k \notin A$ 
This shows that the sequence $\left\{y_{k}\right\}$ is convergent to $x$ with respect to the fuzzy $n$ norm on $X$ such that $y_{k}=x_{k}$ for almost all $k$. conversely, assume that $y_{k}=x_{k}$ for almost all $k$ and $\left(y_{k}, z_{2}, z_{3}, \ldots, z_{n}\right) \longrightarrow^{s t(f-n-N)}\left(x, z_{2}, z_{3}, \ldots, z_{n}\right)$. Let $\epsilon>0$ be given. Then for each $m$, define the set $\left\{k \leq m:\left\|x_{k}-x, z_{2}, z_{3}, \ldots, z_{n}\right\|_{0}^{+} \geq\right.$ $\epsilon\} \subseteq\left\{k \leq m: x_{k} \neq y_{k}\right\} \cup\left\{k \leq m:\left\|y_{k}-x, z_{2}, z_{3}, \ldots, z_{n}\right\|_{0}^{+}>\epsilon\right\}$. Since $\left(y_{k}, z_{2}, z_{3}, \ldots, z_{n}\right) \longrightarrow^{s t(f-n-N)}\left(x, z_{2}, z_{3}, \ldots, z_{n}\right)$, the set $\left\{k \leq m: \| y_{k}-\right.$ $\left.x, z_{2}, z_{3}, \ldots, z_{n} \|_{0}^{+}<\epsilon\right\}$ contains a finite number of elements, say

$$
p=p\left(\epsilon, z_{2}, z_{3}, \ldots, z_{n}\right) .
$$

Therefore

$$
\begin{aligned}
\lim _{m \rightarrow \infty} \frac{1}{m}\left|\left\{k \leq m:\left\|x_{k}-x, z_{2}, z_{3}, \ldots, z_{n}\right\|_{0}^{+} \geq \epsilon\right\}\right| & \\
& \leq \lim _{m \rightarrow \infty} \frac{1}{m}\left|\left\{k \leq m: x_{k} \neq y_{k}\right\}\right|+\lim _{m \rightarrow \infty} \frac{p}{m}=0 .
\end{aligned}
$$

Since $x_{k}=y_{k}$ for almost all $k$. Hence $\left\|x_{k}-x, z_{2}, z_{3}, \ldots, z_{n}\right\|_{0}^{+}<\epsilon$ for almost all $k$. Hence the sequence $\left\{x_{k}\right\}$ is statistically convergent with respect to the fuzzy $n$-norm on $X$.

Theorem 9. Let $(X,\|\bullet, \bullet, \ldots, \bullet\|, L, R)$ be a fuzzy $n$-normed space. Then every statistically convergent sequence $\left\{x_{k}\right\}$ is statistically Cauchy sequence with respect to the fuzzy $n$-norm on $X$.

Proof. Suppose that $\left(x_{k}, z_{2}, z_{3}, \ldots, z_{n}\right) \longrightarrow^{s t(f-n-N)}\left(x, z_{2}, z_{3}, \ldots, z_{n}\right)$. Then for given $\epsilon>0$ we have $\left\|x_{k}-x, z_{2}, z_{3}, \ldots, z_{n}\right\|_{0}^{+}<\frac{\epsilon}{2}$ for a.a.k. Choose $N=N\left(\epsilon, z_{2}, z_{3}, \ldots, z_{n}\right) \in N$ such that $\left\|x_{N\left(\epsilon, z_{2}, z_{3}, \ldots, z_{n}\right)}-x, z_{2}, z_{3}, \ldots, z_{n}\right\|_{0}^{+}<\frac{\epsilon}{2}$. Now $\|\bullet, \bullet, \ldots, \bullet\|_{0}^{+}$being a $n$-norm in the usual sense, we get

$$
\begin{aligned}
& \quad\left\|x_{k}-x_{N\left(\epsilon, z_{2}, z_{3}, \ldots, z_{n}\right)}, z_{2}, z_{3}, \ldots, z_{n}\right\|_{0}^{+} \\
& =\left\|\left(x_{k}-x\right)+\left(x-x_{N\left(\epsilon, z_{2}, z_{3}, \ldots, z_{n}\right)}\right), z_{2}, z_{3}, \ldots, z_{n}\right\|_{0}^{+} \\
& \leq\left\|x_{k}-x, z_{2}, z_{3}, \ldots, z_{n}\right\|_{0}^{+}+\left\|x_{N\left(\epsilon, z_{2}, z_{3}, \ldots, z_{n}\right)}-x, z_{2}, z_{3}, \ldots, z_{n}\right\|_{0}^{+}<\frac{\epsilon}{2}+\frac{\epsilon}{2}=\epsilon,
\end{aligned}
$$

for almost all $k$. Hence $\left\{x_{k}\right\}$ is statistically Cauchy sequence with respect to the fuzzy $n$-norm on $X$

Theorem 10. Let $(X,\|\bullet, \bullet, \ldots, \bullet\|, L, R)$ be a fuzzy $n$-normed space. Let $\left\{x_{k}\right\}$ be a sequence in $X$ and denote $E_{N\left(\epsilon, z_{2}, z_{3}, \ldots, z_{n}\right)}=\left\{k \in N: \| x_{k}-\right.$ $\left.x_{N\left(\epsilon, z_{2}, z_{3}, \ldots, z_{n}\right)}, z_{2}, z_{3}, \ldots, z_{n} \|_{0}^{+} \geq \epsilon\right\}$. If the sequence $\left\{x_{k}\right\}$ is statistically Cauchy sequence, then for every $\epsilon>0, \exists A \subset N$ with $\delta(A)=0$ such that $\| x_{m}-$ $x_{k}, z_{2}, z_{3}, \ldots, z_{n} \|_{0}^{+}<\epsilon \forall m, k \notin A$. 
Proof. For a given $\epsilon>0$, write $A=E_{N\left(\frac{\epsilon}{2}, z_{2}, z_{3}, \ldots, z_{n}\right)}$. Since the sequence $\left\{x_{k}\right\}$ is statistically Cauchy sequence, we can write $\delta(A)=0$. Then for any $m, k \notin A$, we have

$$
\left\|x_{k}-x_{N\left(\epsilon, z_{2}, z_{3}, \ldots, z_{n}\right)}, z_{2}, z_{3}, \ldots, z_{n}\right\|_{0}^{+}<\frac{\epsilon}{2}
$$

and

$$
\left\|x_{m}-x_{N\left(\epsilon, z_{2}, z_{3}, \ldots, z_{n}\right)}, z_{2}, z_{3}, \ldots, z_{n}\right\|_{0}^{+}<\frac{\epsilon}{2} .
$$

Hence $\left\|x_{m}-x_{k}, z_{2}, z_{3}, \ldots, z_{n}\right\|_{0}^{+}<\epsilon, \forall m, k \notin A$.

Definition 11. A fuzzy $n$-norm $|\|\bullet, \bullet, \ldots, \bullet\||$ on a vector space $X$ is said to be fuzzy equivalent to a fuzzy $n$-norm $\|\bullet, \bullet, \ldots, \bullet\|$ written as $|\|\bullet, \bullet, \ldots, \bullet\|| \cong$ $\|\bullet, \bullet, \ldots, \bullet\|$ on $X$ if $\exists \mu, \nu \in L(R)$ and $\mu, \nu \succ \overline{0}$ such that $\forall x \in X$ and every $z_{2}, z_{3}, \ldots, z_{n} \neq 0, z_{2}, z_{3}, \ldots, z_{n} \in X$

$$
\mu \bigotimes\left\|x, z_{2}, z_{3}, \ldots, z_{n}\right\| \preceq\left|\left\|x, z_{2}, z_{3}, \ldots, z_{n}\right\|\right| \preceq \nu \bigotimes\left\|x, z_{2}, z_{3}, \ldots, z_{n}\right\| .
$$

Theorem 12. Let $X$ be a vector space over the real field $R$ and let $\|\bullet, \bullet, \ldots, \bullet\|$ and $|\|\bullet, \bullet, \ldots, \bullet\||$ be fuzzy equivalent fuzzy $n$-norms on $X$. Let $\left\{x_{k}\right\}$ be a sequence in $X$. Then:

(i) $\left\{x_{k}\right\}$ is statistically convergent to $x$ in $(X,\|\bullet, \bullet, \ldots, \bullet\|, L, R)$ if and only if $\left\{x_{k}\right\}$ is statistically convergent to $x$ in $(X,|\|\bullet, \bullet, \ldots, \bullet\||, L, R)$.

(ii) $\left\{x_{k}\right\}$ is statistically Cauchy sequence in $(X,\|\bullet, \bullet, \ldots, \bullet\|, L, R)$ if and only if $\left\{x_{k}\right\}$ is statistically Cauchy sequence in $(X,|\|\bullet, \bullet, \ldots, \bullet\||, L, R)$.

Proof. (i) Let the sequence $\left\{x_{k}\right\}$ be statistically convergent to $x$ in $(X, \| \bullet, \bullet$, $\ldots, \bullet \|, L, R)$. Since $(X,\|\bullet, \bullet, \ldots, \bullet\|, L, R)$ and $(X,|\|\bullet, \bullet, \ldots, \bullet\||, L, R)$ are fuzzy equivalent, $\exists \mu, \nu \in L(R)$ and $\mu, \nu \succ \overline{0}$ such that

$$
\begin{aligned}
\mu \bigotimes\left\|x_{k}-x, z_{2}, z_{3}, \ldots, z_{n}\right\| \preceq \mid \| x_{k}-x, z_{2}, & z_{3}, \ldots, z_{n} \| \mid \\
& \preceq \nu \bigotimes\left\|x_{k}-x, z_{2}, z_{3}, \ldots, z_{n}\right\|,
\end{aligned}
$$

$\forall x_{k}, x \in X$ and $z_{2}, z_{3}, \ldots, z_{n} \in X$. Thus

$$
\begin{aligned}
\mu_{0}^{+}\left\|x_{k}-x, z_{2}, z_{3}, \ldots, z_{n}\right\|_{0}^{+} \leq\left|\left\|x_{k}-x, z_{2}, z_{3}, \ldots, z_{n}\right\|\right|_{0}^{+} & \\
& \leq \nu_{0}^{+}\left\|x_{k}-x, z_{2}, z_{3}, \ldots, z_{n}\right\|_{0}^{+},
\end{aligned}
$$

$\forall k \in N$. By our supposition, we have

$$
s t(f-n-N)-\lim _{k \longrightarrow \infty}\left\|\left(x_{k}-x, z_{2}, z_{3}, \ldots, z_{n}\right)\right\|_{0}^{+}=0 .
$$


Hence

$$
s t(f-n-N)-\lim _{k \rightarrow \infty}\left|\left\|\left(x_{k}-x, z_{2}, z_{3}, \ldots, z_{n}\right)\right\|\right|_{0}^{+}=0
$$

i.e.

$$
\left(x_{k}, z_{2}, z_{3}, \ldots, z_{n}\right) \longrightarrow^{s t(f-n-N)}\left(x, z_{2}, z_{3}, \ldots, z_{n}\right)
$$

in $(X,|\|\bullet, \bullet, \ldots, \bullet\||, L, R)$.

In the same manner if $\left(x_{k}, z_{2}, z_{3}, \ldots, z_{n}\right) \longrightarrow^{s t(f-n-N)}\left(x, z_{2}, z_{3}, \ldots, z_{n}\right)$ in $(X,|\|\bullet, \bullet, \ldots, \bullet\||, L, R)$ then $\left(x_{k}, z_{2}, z_{3}, \ldots, z_{n}\right) \longrightarrow^{s t(f-n-N)}\left(x, z_{2}, z_{3}, \ldots, z_{n}\right)$ in $(X,\|\bullet, \bullet, \ldots, \bullet\|, L, R)$.

(ii) Let the sequence $\left\{x_{k}\right\}$ be statistically Cauchy sequence in $(X,\|\bullet, \bullet, \ldots, \bullet\|$, $L, R)$. Since $(X,\|\bullet, \bullet, \ldots, \bullet\|, L, R)$ and $(X,|\|\bullet, \bullet, \ldots, \bullet\||, L, R)$ are fuzzy equivalent, $\exists \mu, \nu \in L(R)$ and $\mu, \nu \succ \overline{0}$ such that

$$
\mu_{0}^{+}\left\|x_{k}, z_{2}, z_{3}, \ldots, z_{n}\right\|_{0}^{+} \leq\left|\left\|x_{k}, z_{2}, z_{3}, \ldots, z_{n}\right\|\right|_{0}^{+} \leq \nu_{0}^{+}\left\|x_{k}, z_{2}, z_{3}, \ldots, z_{n}\right\|_{0}^{+},
$$

$\forall x \in X$ and $z_{2}, z_{3}, \ldots, z_{n} \in X$. For any $\epsilon>0, \exists N=N\left(\epsilon, z_{2}, z_{3}, \ldots, z_{n}\right) \in N$ such that $\left\|x_{k}-x_{N\left(\epsilon, z_{2}, z_{3}, \ldots, z_{n}\right)}, z_{2}, z_{3}, \ldots, z_{n}\right\|_{0}^{+}<\frac{\epsilon}{\nu_{0}^{+}}$for almost all $k$. Hence

$$
\begin{aligned}
\mid\left\|x_{k}-x_{N\left(\epsilon, z_{2}, z_{3}, \ldots, z_{n}\right)}, z_{2}, z_{3}, \ldots, z_{n}\right\| & \left.\right|_{0} ^{+} \\
& \leq \nu_{0}^{+}\left\|x_{k}-x_{N\left(\epsilon, z_{2}, z_{3}, \ldots, z_{n}\right)}, z_{2}, z_{3}, \ldots, z_{n}\right\|_{0}^{+}<\epsilon,
\end{aligned}
$$

for a.a.k. Therefore the sequence $\left\{x_{k}\right\}$ is statistically Cauchy sequence in $(X,|\|\bullet, \bullet, \ldots, \bullet\||, L, R)$. In the same manner if $\left\{x_{k}\right\}$ is statistically Cauchy sequence in $(X,|\|\bullet, \bullet, \ldots, \bullet\||, L, R)$ then it is statistically Cauchy sequence in $(X,\|\bullet, \bullet, \ldots, \bullet\|, L, R)$.

\section{Statistical Limit Point and Statistical Cluster Point in Fuzzy $\boldsymbol{n}$-Normed Spaces}

Statistical limit point of sequence $\left\{x_{k}\right\}$ has been define and studied by Fridy[14] and for fuzzy number by Aytar[9]. In this section, we define the notions of thin subsequence, non-thin subsequence, statistical limit point and statistical cluster point in fuzzy $n$-normed space.

Definition 13. Let $(X,\|\bullet, \bullet, \ldots, \bullet\|, L, R)$ be fuzzy $n$-normed space and $\left(x_{k}\right)$ be a sequence in $(X,\|\bullet, \bullet, \ldots, \bullet\|, L, R)$. An element $x \in X$ is said to be a limit point of the sequence $\left(x_{k}\right)$ if there is a subsequence of $\left(x_{k}\right)$ which converges to $x$ with respect to the fuzzy $n$-norm on $X$. The set of all limit points of the sequence $\left(x_{k}\right)$ is denoted by $L_{(f-n-N)}\left(x_{k}\right)$. 
Definition 14. Let $\left(x_{k}\right)$ be a sequence in fuzzy $n$-normed space $(X, \| \bullet, \bullet$, $\ldots, \bullet \|, L, R)$ and $\left(x_{k_{j}}\right)$ be a subsequence of $\left(x_{k}\right)$. Write $K=\left\{k_{j}: j \in N\right\}$. If $\delta(K)=0$ then we say that $\left(x_{k_{j}}\right)$ is a thin subsequence of $\left(x_{k}\right)$. A subsequence $\left(x_{k_{j}}\right)$ is said to be a non-thin subsequence of $\left(x_{k}\right)$ if $\delta(K)>0$ or $\delta(K)$ does not exists, namely, $\bar{\delta}(K)>0$

Definition 15. Let $(X,\|\bullet, \bullet, \ldots, \bullet\|, L, R)$ be fuzzy $n$-normed space and $\left(x_{k}\right)$ be a sequence in $(X,\|\bullet, \bullet, \ldots, \bullet\|, L, R)$. An element $x \in X$ is said to be statistical limit point of the sequence $\left(x_{k}\right)$ if $\exists$ a non-thin subsequence of $\left(x_{k}\right)$ which converges to $x$ with respect to the fuzzy $n$-norm on $X$. The set of all statistical limit points of the sequence $\left(x_{k}\right)$ is denoted by $S L_{(f-n-N)}\left(x_{k}\right)$.

Definition 16. Let $(X,\|\bullet, \bullet, \ldots, \bullet\|, L, R)$ be fuzzy $n$-normed space and $\left(x_{k}\right)$ be a sequence in $(X,\|\bullet, \bullet, \ldots, \bullet\|, L, R)$. An element $x \in X$ is said to be statistical cluster point of the sequence $\left(x_{k}\right)$ with respect to the fuzzy $n$-norm on $X$ if for every $\epsilon>0$ and $z_{2}, z_{3}, \ldots, z_{n} \in X, \bar{\delta}\left(\left\{k \in N:\left\|x_{k}-x, z_{2}, z_{3}, \ldots, z_{n}\right\|_{0}^{+}<\right.\right.$ $\epsilon\})>0$. The set of all statistical cluster points of the sequence $\left(x_{k}\right)$ is denoted by $S C_{(f-n-N)}\left(x_{k}\right)$.

Remark 17. An element $x \in S C_{(f-n-N)}\left(x_{k}\right)$ implies that $\bar{\delta}(\{k \in N$ : $\left.\left.\left\|x_{k}-x, z_{2}, z_{3}, \ldots, z_{n}\right\|_{\alpha}^{+}<\epsilon\right\}\right)>0$ and $\bar{\delta}\left(\left\{k \in N:\left\|x_{k}-x, z_{2}, z_{3}, \ldots, z_{n}\right\|_{\alpha}^{-}<\right.\right.$ $\epsilon\})>0 \forall \epsilon>0, \alpha \in[0,1]$ and $z_{2}, z_{3}, \ldots, z_{n} \in X$.

Theorem 18. Let $(X,\|\bullet, \bullet, \ldots, \bullet\|, L, R)$ be fuzzy $n$-normed space. Then for every sequence $\left(x_{k}\right)$ in $X$, we have $S L_{(f-n-N)}\left(x_{k}\right) \subseteq S C_{(f-n-N)}\left(x_{k}\right) \subseteq$ $L_{(f-n-N)}\left(x_{k}\right)$.

Proof. Let $x \in S L_{(f-n-N)}\left(x_{k}\right)$. Then $\exists$ a non-thin sub sequence $\left(x_{k_{j}}\right)$ of the sequence $\left(x_{k}\right)$ which converges to $x$, namely, $\bar{\delta}\left(\left\{k_{j}: j \in N\right\}\right)=d>0$. Since $\left\{k \in N:\left\|x_{k}-x, z_{2}, z_{3}, \ldots, z_{n}\right\|_{0}^{+}<\epsilon\right\} \supseteq\left\{k_{j} \in N:\left\|x_{k_{j}}-x, z_{2}, z_{3}, \ldots, z_{n}\right\|_{0}^{+}<\right.$ $\epsilon\}$ for every $\epsilon>0$ and so $\left\{k \in N:\left\|x_{k}-x, z_{2}, z_{3}, \ldots, z_{n}\right\|_{0}^{+}<\epsilon\right\} \supseteq\left\{k_{j}: j \in\right.$ $N\} \backslash\left\{k_{j} \in N:\left\|x_{k_{j}}-x, z_{2}, z_{3}, \ldots, z_{n}\right\|_{0}^{+} \geq \epsilon\right\}$. Since

$$
\left(x_{k_{j}}, z_{2}, z_{3}, \ldots, z_{n}\right) \longrightarrow^{s t(f-n-N)}\left(x, z_{2}, z_{3}, \ldots, z_{n}\right),
$$

the set $\left\{k_{j} \in N:\left\|x_{k_{j}}-x, z_{2}, z_{3}, \ldots, z_{n}\right\|_{0}^{+} \geq \epsilon\right\}$ is finite for any $\epsilon>0$, hence we have $\bar{\delta}\left(\left\{k \in N:\left\|x_{k}-x, z_{2}, z_{3}, \ldots, z_{n}\right\|_{0}^{+}<\epsilon\right\}\right) \geq \bar{\delta}\left(\left\{k_{j}: j \in N\right\}\right)-$ $\bar{\delta}\left(\left\{k_{j} \in N:\left\|x_{k_{j}}-x, z_{2}, z_{3}, \ldots, z_{n}\right\|_{0}^{+} \geq \epsilon\right\}\right)=d>0$. Thus, for any $\epsilon>0$ and $z_{2}, z_{3}, \ldots, z_{n} \in X, \bar{\delta}\left(\left\{k \in N:\left\|x_{k}-x, z_{2}, z_{3}, \ldots, z_{n}\right\|_{0}^{+}<\epsilon\right\}\right)>0$, i.e., $x \in S C_{(f-n-N)}\left(x_{k}\right)$.

Let $x \in S C_{(f-n-N)}\left(x_{k}\right)$. For every $\epsilon>0$ and $z_{2}, z_{3}, \ldots, z_{n} \in X$, write $\bar{\delta}\left(\left\{k \in N:\left\|x_{k}-x, z_{2}, z_{3}, \ldots, z_{n}\right\|_{0}^{+}<\epsilon\right\}\right)>0$ which gives every $(\epsilon, 0)$ - 
neighborhood of $x$ contains infinitely many terms of the sequence $\left(x_{k}\right)$. i.e., $x \in$ $L_{(f-n-N)}\left(x_{k}\right)$. Therefore $S L_{(f-n-N)}\left(x_{k}\right) \subseteq S C_{(f-n-N)}\left(x_{k}\right) \subseteq L_{(f-n-N)}\left(x_{k}\right)$.

Theorem 19. Let $(X,\|\bullet, \bullet, \ldots, \bullet\|, L, R)$ be fuzzy $n$-normed space and $\left(x_{k}\right)$ be a sequence in $(X,\|\bullet, \bullet, \ldots, \bullet\|, L, R)$. Then

$$
S L_{(f-n-N)}\left(x_{k}\right)=S C_{(f-n-N)}\left(x_{k}\right)=\{x\},
$$

if $\left(x_{k}, z_{2}, z_{3}, \ldots, z_{n}\right) \longrightarrow^{s t(f-n-N)}\left(x, z_{2}, z_{3}, \ldots, z_{n}\right)$.

Proof. Let $\left(x_{k}, z_{2}, z_{3}, \ldots, z_{n}\right) \longrightarrow^{s t(f-n-N)}\left(x, z_{2}, z_{3}, \ldots, z_{n}\right)$. Therefore $x \in S C_{(f-n-N)}\left(x_{k}\right)$. Let us assume that $\exists$ at least one $y \in \operatorname{SC}_{(f-n-N)}\left(x_{k}\right)$ such that $y \neq x$. For every $\epsilon>0$ and every $z_{2}, z_{3}, \ldots, z_{n} \neq 0, z_{2}, z_{3}, \ldots, z_{n} \in$ $X$ such that $\left\{k \in N:\left\|x_{k}-x, z_{2}, z_{3}, \ldots, z_{n}\right\|_{0}^{+} \geq \epsilon\right\} \supseteq\left\{k \in N: \| x_{k}-\right.$ $\left.y, z_{2}, z_{3}, \ldots, z_{n} \|_{0}^{+}<\epsilon\right\}$ is true. Therefore $\bar{\delta}\left(\left\{k \in N:\left\|x_{k}-x, z_{2}, z_{3}, \ldots, z_{n}\right\|_{0}^{+} \geq\right.\right.$ $\epsilon\}) \geq \bar{\delta}\left(\left\{k \in N:\left\|x_{k}-y, z_{2}, z_{3}, \ldots, z_{n}\right\|_{0}^{+}<\epsilon\right\}\right)$. Since

$$
\left(x_{k}, z_{2}, z_{3}, \ldots, z_{n}\right) \longrightarrow^{s t(f-n-N)}\left(x, z_{2}, z_{3}, \ldots, z_{n}\right)
$$

we have

$$
\delta\left(\left\{k \in N:\left\|x_{k}-x, z_{2}, z_{3}, \ldots, z_{n}\right\|_{0}^{+} \geq \epsilon\right\}\right)=0 .
$$

This gives $\bar{\delta}\left(\left\{k \in N:\left\|x_{k}-x, z_{2}, z_{3}, \ldots, z_{n}\right\|_{0}^{+} \geq \epsilon\right\}\right)=0$. Thus

$$
\bar{\delta}\left(\left\{k \in N:\left\|x_{k}-y, z_{2}, z_{3}, \ldots, z_{n}\right\|_{0}^{+}<\epsilon\right\}\right)=0 .
$$

This is a contradiction to our assumption $y \in S C_{(f-n-N)}\left(x_{k}\right)$. Therefore $S C_{(f-n-N)}\left(x_{k}\right)=\{x\}$. But from hypothesis $\left(x_{k}, z_{2}, z_{3}, \ldots, z_{n}\right) \longrightarrow^{s t(f-n-N)}$ $\left(x, z_{2}, z_{3}, \ldots, z_{n}\right)$. By Theorem 8 and definition 15 , we get $x \in S L_{(f-n-N)}\left(x_{k}\right)$.

Now Theorem 18 yields $S L_{(f-n-N)}\left(x_{k}\right)=S C_{(f-n-N)}\left(x_{k}\right)=\{x\}$.

\section{References}

[1] T. Bag, S.K. Samanta, Finite dimensional fuzzy normed linear spaces, Journal of fuzzy mathematics, 11, No. 3 (2003), 687-705.

[2] F. Nuray, E. Savas, statistical convergence of sequences of fuzzy numbers, Math. Slovoca, 45 (1995), 269-273.

[3] J.A. Fridy, On statistical convergence, Analysis, 5 (1985), 301-313. http://dx.doi.org/10.1524/anly.1985.5.4.301. 
[4] C. Sencimen, S. Pehlivan, Statistical convergence in fuzzy normed linear spaces, Fuzzy sets and Systems, 159 (2008), 361-370. http://dx.doi.org/10.1016/j.fss.2007.06.008.

[5] M. Mizumoto, J. Tanaka, Some properties of fuzzy numbers, in:M.M.Gupta et al.(Eds.), Advances in fuzzy set theory and Applications, North-Holland, Amsterdam (1979).

[6] M. Gurdal, S. Pehlivan, Statistical convergence in 2-normed spaces, Southeast asian bulletin of mathematics, 33 (2009), 257-264.

[7] H. Steinhaus, Sur la convergence ordinaire et la convergence asymptotique, Colloq. Math., 2 (1951), 73-74.

[8] A.R Freedman, J.J. Sember, Densities and summability, Pacific J. Math., 95 (1981), 293-305. http://dx.doi.org/10.2140/pjm.1981.95.293

[9] S. Aytar, Statistical limit point of sequences of fuzzy numbers, Inform. Sci., 165 (2004), 129-138. http://dx.doi.org/10.1016/j.ins.2003.06.003.

[10] B.Surender Reddy, Statistical convergence in n-normed spaces, International Mathematical Forum, 5, No. 24 (2010), 1185-1193.

[11] H.Fast, Sur la convergence statistique, Colloq. Math. 2 (1951), 241-244.

[12] S.A. Mohiuddine, H. Sevli, M. Cancan, Statistical convergence in fuzzy 2-normed space, Journal of Computational analysis and applications, 12, No. 4 (2010), 787-798.

[13] B.C. Tripathy, On statistically convergent sequences, Bull. Calcutta Math. Soc., 90 (1998), 259-262.

[14] J.A. Fridy, Statistical limit points, Proc. Amer. Math. Soc., 118 (1993), 1187-1192. http://dx.doi.org/10.1090/S0002-9939-1993-1181163-6

[15] A.K. Katsaras, Fuzzy topological vector spaces, Fuzzy sets and systems, 12 (1984), 143-154. http://dx.doi.org/10.1016/0165-0114(84)90034-4

[16] O. Kaleva, S. Seikkala, On fuzzy metric spaces, Fuzzy sets and systems, 12 (1984), 215-229. http://dx.doi.org/10.1016/0165-0114(84)90069-1

[17] Felbin.C, Finite dimensional fuzzy normed linear spaces, Fuzzy sets and systems, 48 (1992), 239-248. http://dx.doi.org/10.1016/01650114(92)90338-5 
[18] A.L. Narayan and S. Vijayabalaji, Fuzzy n-normed linear space, International journal of mathematics and mathematical sciences, 24 (2005), 3963-3977. http://dx.doi.org/10.1155/IJMMS.2005.3963 